\title{
Advantages and technical issues of regenerative brake method at all over the speed range
}

\author{
Febry Pandu WIJAYA*, Sho WATANABE**, Tatsuhito SAITO*, Hiroyasu KOBAYASHI* \\ and Keiichiro KONDO* \\ ${ }^{*}$ Graduate School of Engineering, Chiba University \\ 1-33 Yayoi-cho, Inage-ku, Chiba-shi, Chiba 263-8522, Japan \\ E-mail: fpwijaya@chiba-u.jp \\ ${ }^{* \star}$ Odakyu Electric Railway Co. Ltd \\ 9-25-2 Kitami, Setagaya-ku, Tokyo 157-0067, Japan
}

Received: 8 August 2016; Revised: 24 October 2016; Accepted: 13 December 2016

\begin{abstract}
In this paper, the authors propose "regenerative brake notch" method which follows the maximum regenerative brake force according to the vehicle speed. This specially designed brake force reference selected by the train driver aims at saving the kinetic energy which originally would be dissipated by the mechanical brake in the high speed range. By means of the regenerative brake notch, it is able to save much kinetic energy, but the brake section is slightly prolonged. Another drawback of this method is how to inform the starting braking point to the driver, because this brake system does not generate constant regenerative brake force all over the speed range. To cope with this problem, the authors propose the utilization of imaginary train that run along with the real train in combination with the preinstalled train information control system (TICS) to obtain the correct starting braking point and inform to the driver. The running profiles of these trains are compared to obtain the correct starting braking point. By means of this method, only the point to start braking is required and the driver can regulate the brake force in the lower speed range to stop the train at the station. In this paper, the energy saving effect by the regenerative brake notch is compared with the conventional constant deceleration brake notch in terms of running distance, error of the starting braking point and rail adhesion coefficient. Through the calculation study, the energy saving effect and usefulness of the proposed regenerative brake notch is revealed.
\end{abstract}

Key words : Regenerative brake, Energy saving, Adhesion coefficient, Less modification of the vehicle, No assistance from ground facility

\section{Introduction}

In the DC-electrified railway system, there are various possibilities to reduce the energy consumption of the train, for instance saving the regeneration energy to the energy storage devices (ESDs) either onboard (Ogihara et al., 2011) or wayside (Suzuki et al., 2016) and the use of pure regenerative brake to stop the train (Koseki and Noda, 2010). The energy consumption of ESDs-train can be reduced by the use of regenerative energy for powering phase. However, this technology requires additional equipment with high initial cost. The pure regenerative brake has a merit due to the use of inheritance features of traction motor; hence no additional equipment is required. The principle to save the energy is to use the regenerative brake only to stop the train and avoiding the use of mechanical brake as much as possible. This paper discusses the advantages and technical issues of the pure regenerative brake method.

In the brake control of Electric Motive Units, the constant brake force that combines regenerative and mechanical brake forces is commonly applied. The constant brake force reference is generated according to the driver's command in the cabin, e.g. from notch 1 to 7 , where higher number means stronger brake. Since the traction motor power is limited by the inverter voltage and current, hence the regenerative brake force in the high speed range is limited. In this condition, the mechanical brake force compensates the shortage of the regenerative brake force to obtain constant deceleration. Thus, much kinetic energy is dissipated by the stronger mechanical brake in the higher speed range which 
leads to more heat generation and wear of the mechanical brake parts. We can expect to save the kinetic energy and to reduce the wear of mechanical brake parts, if the weaker brake force is applied in the higher speed range in accordance with the regenerative brake torque versus speed characteristics of the traction motors.

One of the measures to achieve weaker brake force in the higher speed range and stronger brake force in the lower speed range is to inform the driver to apply brake notch with lower number in the higher speed range and higher number in the lower speed range based on pre-calculated running reference curves and train position measured by GPS (Watanabe et al., 2014). This measure is called power limited brake. In this system, the driver is informed which brake notch to be selected by the train operation assistance system which acquires the train position and speed information from GPS. This method has a great benefit where no modification of the vehicle is required. However, the drawback is that the regenerative brake force is only applied by the step and always less than the maximum regenerative brake performance. Another drawback is that duty of the driver becomes heavy to follow the instruction to apply certain brake notch based on position and speed measured by GPS.

In this paper, the authors propose "regenerative brake notch" method which follows the maximum regenerative brake force according to vehicle speed. The benefit of this system is always utilizing the maximum regenerative brake force featured by the traction motor characteristics. In this method, the driver only applies the regenerative brake notch one time and will not be instructed to change the brake notch until the vehicle speed reduces to lower speed range. Since the regenerative brake notch does not generate constant deceleration all over the speed range, hence it is difficult to find the starting braking point and inform to the driver. There will be a lot of storing data in the controller that is calculated offline using motion equation by comparing each combination of train speed and distance because the initial braking speed varies according to the track conditions, such as gradient, curve, speed limit and adhesion coefficient. In other words, the calculation load of the controller is high.

To cope with this problem, the authors propose the utilization of imaginary train in combination with the preinstalled train information control system (TICS) to obtain the correct starting braking point and inform to the driver. The calculation is done onboard through TICS by making an imaginary train that run along with the real train by considering the track conditions. The purpose of imaginary train is to estimate the braking pattern of the real train that is calculated beforehand in the powering phase of the real train. In other words, the powering phase of the imaginary train represents the braking characteristics of the real train under the condition of regenerative brake notch. The running profiles of these trains are then compared to obtain the correct starting braking point. By means of this method, only the point to start braking is required and the driver can regulate the brake force in the lower speed range to stop the train at the station if required. The energy saving effect by the regenerative brake notch is compared with the conventional constant deceleration brake notch in terms of running distance, error of the starting braking point and rail adhesion coefficient. Through the calculation study, the energy saving effect and usefulness of the proposed regenerative brake notch is revealed.

\section{Basic features of the regenerative brake notch}

In this chapter, the basic characteristics of the regenerative brake notch is studied and compared with the constant deceleration brake notch under the conditions of the assumed vehicle specifications given in Table 1 and the assumed powering and regenerating brake forces shown in Fig. 1. The deceleration rate for the regenerative brake notch is higher than the conventional one in the low speed range, where its maximum value is $-3.0 \mathrm{~km} / \mathrm{h} / \mathrm{s}$. To obtain the same running time for both brake systems, the deceleration rate for the conventional brake notch is assumed as $-2.5 \mathrm{~km} / \mathrm{h} / \mathrm{s}$. The maximum deceleration rate for the conventional brake notch itself is assumed as $-3.0 \mathrm{~km} / \mathrm{h} / \mathrm{s}$ in this study.

As a comparison example, Fig. 2 shows vehicle running profiles with the regenerative brake notch and the constant deceleration brake notch under the conditions of equivalent running distance and maximum vehicle speed. In Fig. 2(a), the constant deceleration brake is applied at $450 \mathrm{~m}$ in advance to the station, whereas the regenerative brake notch is applied at $750 \mathrm{~m}$ in advance to the station. In this condition, the regenerative brake notch requires $300 \mathrm{~m}$ longer brake section. Likewise, the regenerative brake notch requires $10 \mathrm{~s}$ longer brake duration as shown in Fig. 2(b). In addition, the brake force of regenerative brake notch is stronger than the conventional one in low speed range. Thus, due to longer brake duration and stronger brake force in low speed range, more kinetic energy can be recovered by regenerative brake notch method which is shown in Fig. 3. 
Table 1 Specifications of the assumed vehicle.

\begin{tabular}{l|cl}
\hline Number of car & 10 & \\
Total weight & 425 & $\mathrm{t}$ \\
Starting acceleration & 3.0 & $\mathrm{~km} / \mathrm{h} / \mathrm{s}$ \\
Maximum speed & 95 & $\mathrm{~km} / \mathrm{h}$ \\
Constant torque region & $0-35$ & $\mathrm{~km} / \mathrm{h}$ \\
Constant power region & $35-65$ & $\mathrm{~km} / \mathrm{h}$ \\
Characteristics region & $65-$ & $\mathrm{km} / \mathrm{h}$ \\
Auxiliary power & 30 & $\mathrm{~kW} / \mathrm{car}$ \\
\hline
\end{tabular}

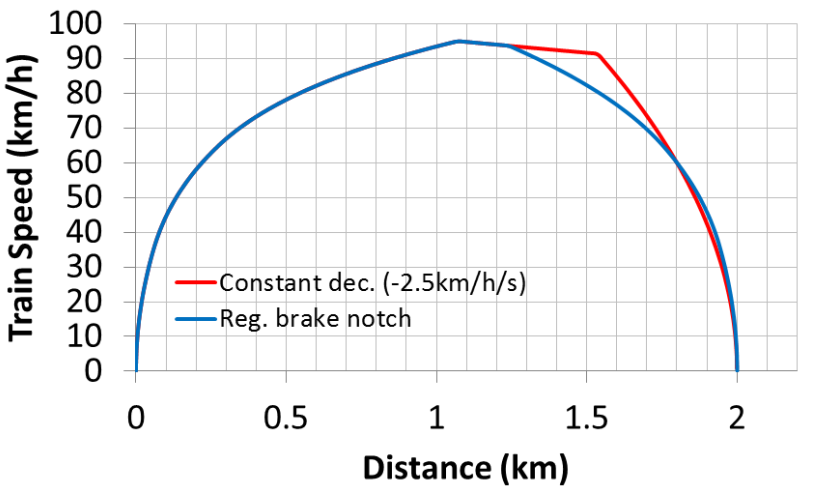

(a) Speed vs. distance

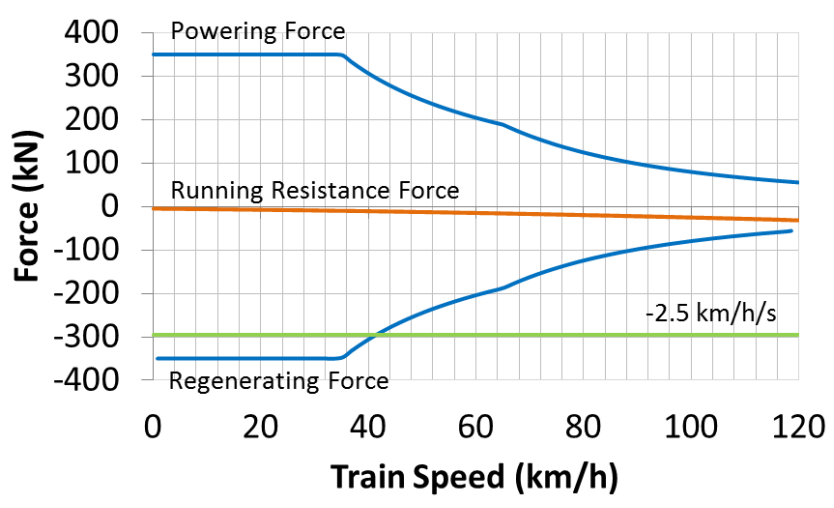

Fig. 1. Powering force and regenerating force characteristics of the assumed vehicle.

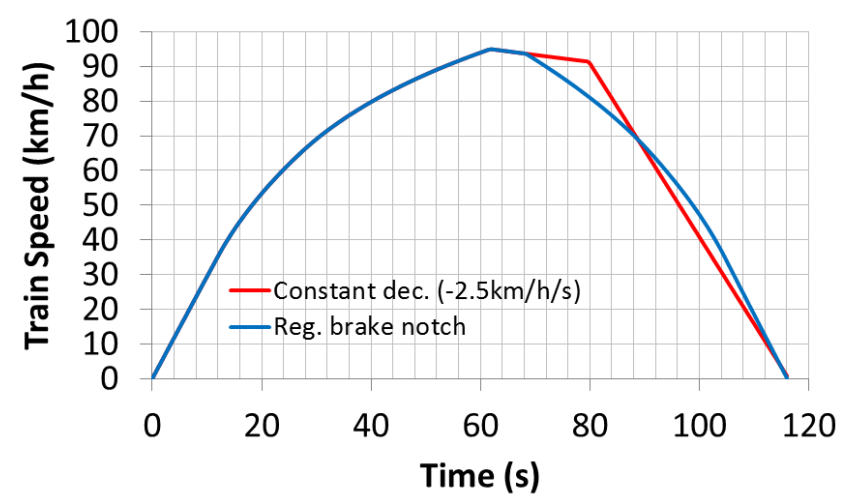

(b) Speed vs. time

Fig. 2. Comparison of the vehicle speed profiles between the regenerative brake notch and the conventional constant deceleration brake notch.

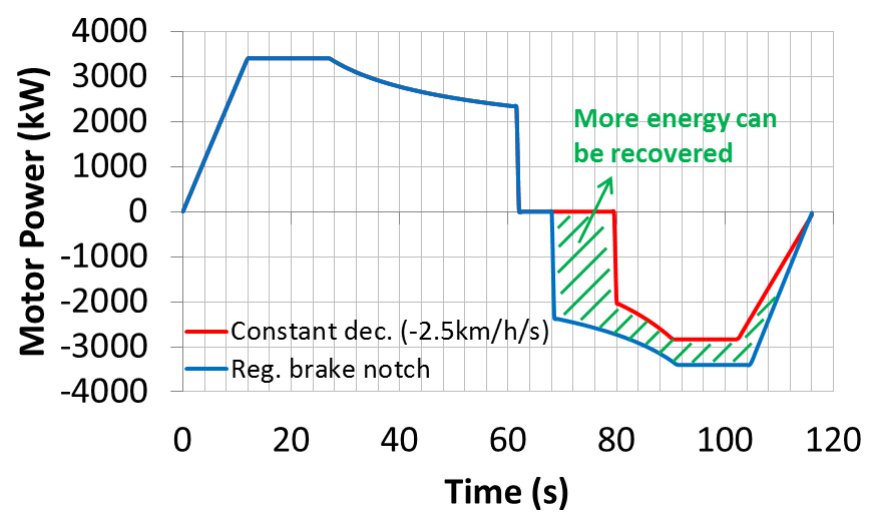

Fig. 3. The traction motor power of the regenerative brake notch and the constant deceleration brake notch.

These comparisons reveal that the regenerative brake notch requires more experience of the driver to stop the train within designated running distance because of longer brake duration and not constant deceleration rate all over the speed range. Therefore, some kind of assistance or guidance is required to notify the driver where the correct starting braking point is. To meet this requirement, the authors propose that the driver is assisted by the train location information obtained by the preinstalled TICS. By means of this method, only the point to start braking is required and the driver can regulate the brake force in the lower speed range to stop the train at the station if required.

Figure 4 shows the system block diagram of the regenerative brake notch system. In order to operate the regenerative brake notch in the practical situation, a new button called "regenerative brake notch button" representing the functionality of regenerative brake notch system can be installed in the driver cabin. Generally, the brake handle is used for constant deceleration brake by combining regenerative brake and mechanical brake. By adding this 
regenerative brake notch button with normally open state, the driver can apply the regenerative brake notch by pushing that button one time and followed by operating the brake handle. If the regenerative brake notch cannot be operated properly to stop the train, for example due to delayed brake point, slippery condition or the estimated stopping distance can only be covered by the constant deceleration brake; the driver may apply the constant deceleration brake method. Afterward, the brake operation will be continued with constant deceleration brake method. Furthermore, in this system, the driver has full responsibility to secure the train stopping position, either by applying regenerative brake notch or constant deceleration brake method.

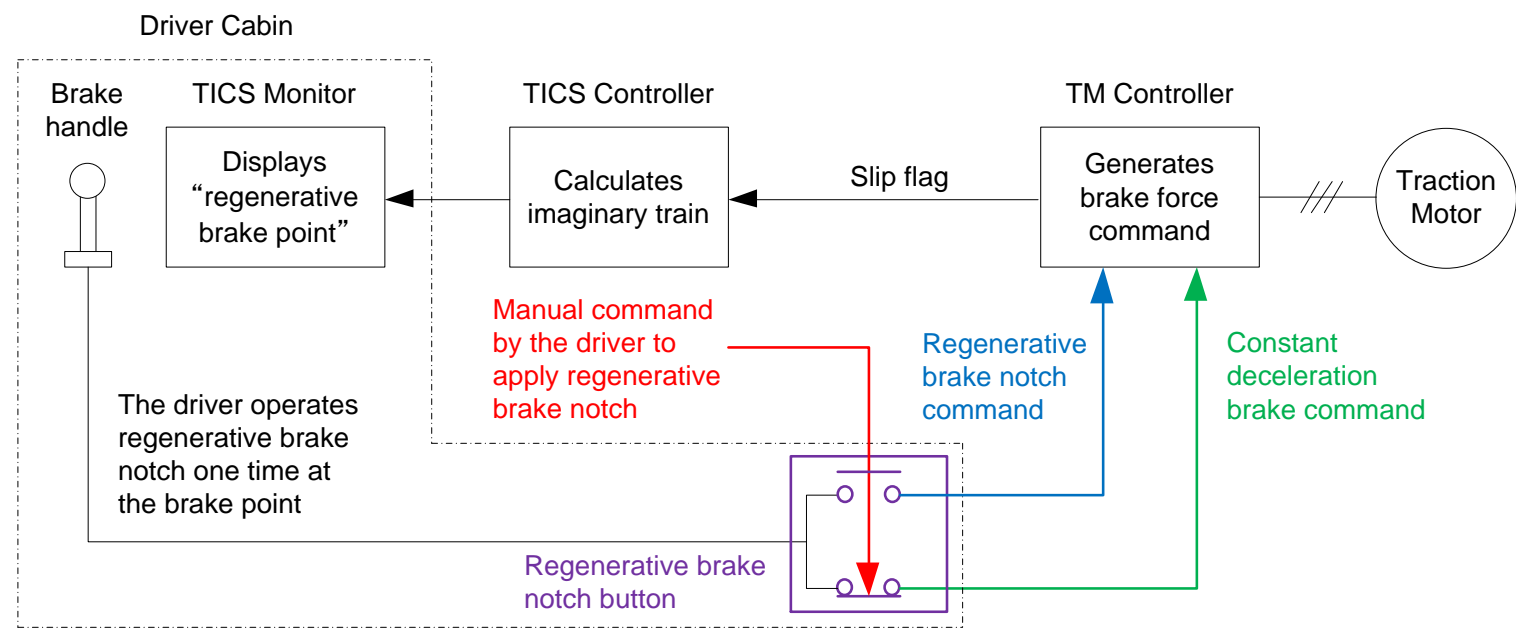

Fig. 4. The system block diagram that shows the tasks of TICS controller, TM (traction motor) controller and the driver to operate the regenerative brake notch.

\section{Driver assisting method based on TICS}

In the power limited brake method (Watanabe et al., 2014), a driving assistance screen is attached in the cabin to notify the driver which brake notch is to be selected actively. The notch selection is determined by actively referring to the pre-calculated (off line calculation) vehicle running profile based on the vehicle position obtained from GPS and the vehicle speed measured optically from the onboard speed meter. This method features no need to install any special control function on the vehicle. All of the driver assistance equipment, such as onboard screen, onboard CCD camera and onboard computer system to follow the train running profile, can be attached on the existing vehicles. One of the benefits of this system is suitable for retrofitting the existing vehicles at low cost.

On the other hand, our proposed system is another solution to recover more kinetic energy without major change of the vehicles or the ground facilities, such as Automatic Train Operation (ATO) system. The proposed system aims at recently build inverter-fed AC traction motor system with TICS. As far as these systems are equipped, the retrofit is possible. In this system, the authors propose the utilization of TICS to calculate the correct starting braking point to be informed to the driver. The calculation is done onboard through TICS by making an imaginary train that run along with the real train, which will be explained later on.

The braking distance of the train when applying regenerative brake notch system can be calculated onboard using motion equation. However, since the regenerating brake and running resistance forces have nonlinear relationship with the train speed as shown in Fig. 1, there will be a lot of storing data in the controller that is calculated offline by comparing each combination of train speed and distance because the initial braking speed varies according to the track conditions, such as gradient, curve, speed limit and adhesion coefficient. In other words, the calculation load of the controller is high. In order to reduce the calculation load in the controller, this paper uses imaginary train that takes into account the track conditions which can be obtained from TICS.

The explanations of the imaginary train are given in the flowchart of Fig. 5 with the following description. The purpose of imaginary train is to estimate the braking pattern of the real train that is calculated beforehand in the powering phase of the real train. In other words, the powering phase of the imaginary train represents the braking characteristics of the real train under the condition of regenerative brake notch as shown in Fig. 6(a). In the former half part of running distance, the imaginary train is generated by TICS under the same sampling time with the real train using the following discrete equation. 


$$
v\left(t_{0}+d t\right)^{2}-v\left(t_{0}\right)^{2}=2 \cdot\left(\frac{F_{\text {regenerating }}\left(t_{0}\right)-F_{\text {resistance }}\left(t_{0}\right)}{m}\right) \cdot s\left(t_{0}+d t\right)
$$

where $d t$ is the sampling time, $t_{0}$ is the initial time, $v\left(\mathrm{t}_{0}\right)$ is the initial speed, $v\left(\mathrm{t}_{0}+\mathrm{dt}\right)$ is the speed after $d t, s\left(\mathrm{t}_{0}+\mathrm{dt}\right)$ is the running distance at each $d t, F_{\text {regenerating }}\left(\mathrm{t}_{0}\right)$ is the regenerating force of the traction motor, and $F_{\text {resistance }}\left(\mathrm{t}_{0}\right)$ is the running resistance force including gradient and curve. Equation (1) models the change of kinetic energy within short period of $d t$, such that $F_{\text {regenerating }}$ and $F_{\text {resistance }}$ are regarded as constant values because the train speed does not change so much. The running resistance force $F_{\text {resistance }}$ uses minus sign because it represents the braking characteristics of the real train. In addition, this system is assumed to be used for commuter train where much regenerative loads are expected. Hence,

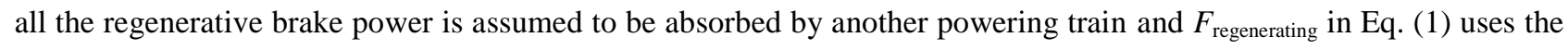
motor characteristics only. Furthermore, when the train is running at the early morning, at late night or at light load condition, the driver may judge to apply the constant deceleration brake to stop the train if the regenerative brake notch force is not enough.

In this case, the track conditions of the real train do not affect the running profile of the imaginary train because it only uses the same sampling time. The imaginary train will stop its operation when the speed reaches the maximum allowable speed in the track, e.g. $95 \mathrm{~km} / \mathrm{h}$ in this study. At this point, the estimated braking pattern of the real train is completely generated. The maximum speed of the track is considered, because the initial braking speed will not exceed this value. Thus, the maximum braking distance $S_{\max }$ is obtained as well. In the latter part of running distance, when the remaining running distance of the real train is equal to $S_{\max }$, the stored pattern of the imaginary train is reflected to output the imaginary line representing the braking pattern of the real train. The running profiles of these trains are then compared to obtain the correct starting braking point $Z$ in Fig. 6(b). Please note that $S_{\max }$ is not always same with the starting braking point $Z$, because $S_{\max }$ represents the maximum braking distance when the initial braking speed is the maximum speed, where the initial braking speed is not always same with the maximum speed.

In addition to the results shown in Fig. 6 which is applied to the flat track and same maximum speed, the imaginary train can also be applied to different track gradients and different track speed limits as depicted in Figs. 7(a) and 7(b). From these results, it can be seen that the imaginary train estimates the condition in the braking part of the real train, without being affected by the condition in the powering part of the real train. In other words, as long as the information in the latter half part of the running distance is obtained beforehand, the imaginary train will be able to estimate the correct starting braking point during powering phase of the real train. Therefore, from the results in Figs. 6 and 7, the imaginary train is sufficiently effective to find the starting braking point and applying regenerative brake notch under several different conditions.

The vehicle speed and position information are obtained from navigation function of TICS. The accuracy of these information are within several meters and few $\mathrm{km} / \mathrm{h}$. These are equivalent with or more accurate than the position and the speed information obtained from GPS. The accuracy of the position does not affect the performance of the train speed control because only the starting braking point is required in the proposed regenerative brake notch method. In this system, the driver is notified by the TICS several seconds in advance to the correct starting braking point. The major factor of the error to start the regenerative brake notch may be delay of the driver's manipulation. For example, assuming the train speed is $90 \mathrm{~km} / \mathrm{h}$, one second delay of the driver's manipulation causes $25 \mathrm{~m}$ error of the starting braking point. This error can be compensated by the driver during the train deceleration by selecting the conventional constant deceleration brake handle. Thus, the accuracy of the train stopping position at the station is secured by the driver's brake handle manipulation.

The same principle can be expected in other cases, such as in the steep grade section where the load condition is larger that requires mechanical brake compensation by the driver. Another case is when the powering train is less or far from the braking train. Since the regenerative brake force is limited, the mechanical brake will assist the regenerative brake notch to stop the train. In the case of the lower adhesive coefficient, such as rainy or snowy weather, wheel skid is expected especially in the case of applying stronger brake in the low speed range. Under this condition, the driver may apply the constant deceleration brake notch instead of the regenerative brake notch to secure the train stopping position. However, this paper reveals that under such condition, even the regenerative brake notch can be applied solely to stop the train at the slippery rail conditions, which will be described in chapter 4 . 


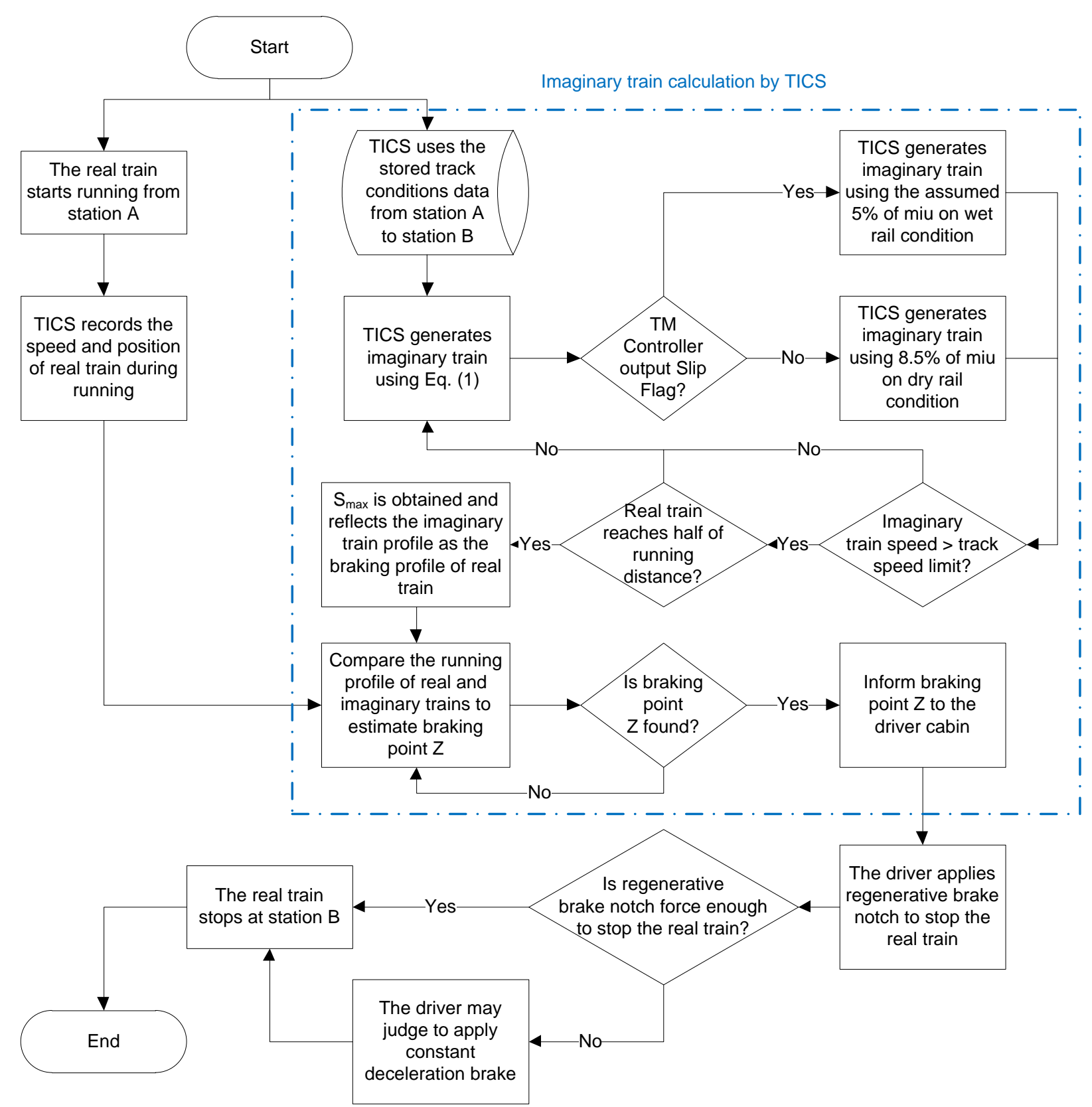

Fig. 5. Flowchart of the imaginary train calculation by TICS along with the operation of the real train.

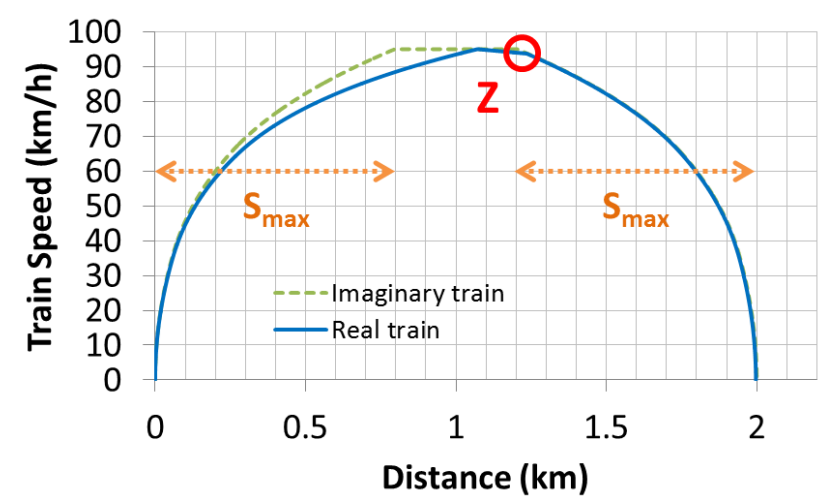

(a) Speed vs. distance

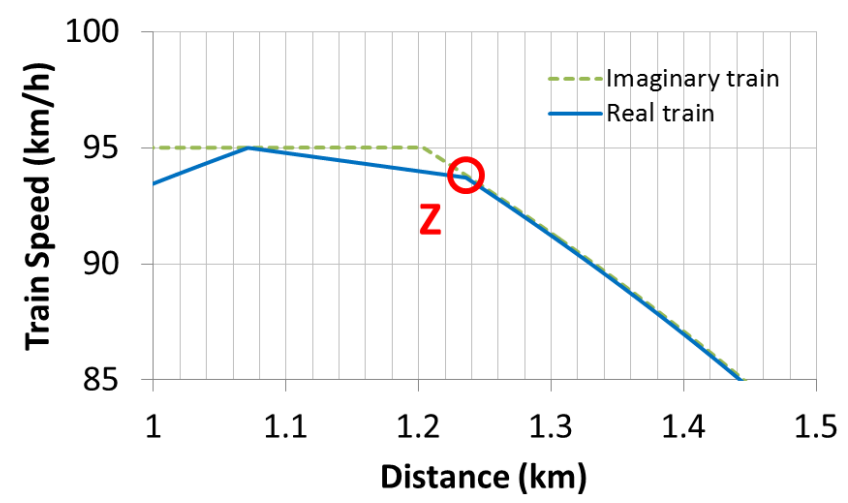

(b) The correct starting braking point $\mathrm{Z}$ at Fig. 6(a)

Fig. 6. The proposed method to find the correct starting braking point using the imaginary train. 


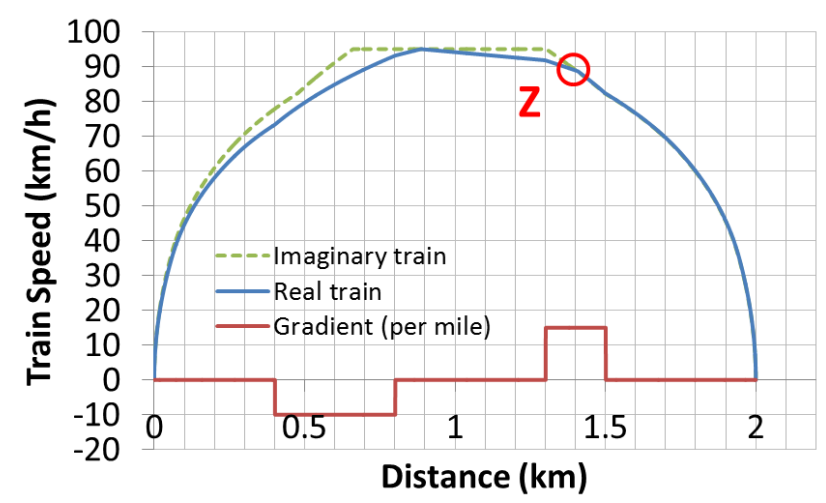

(a) Different track gradients

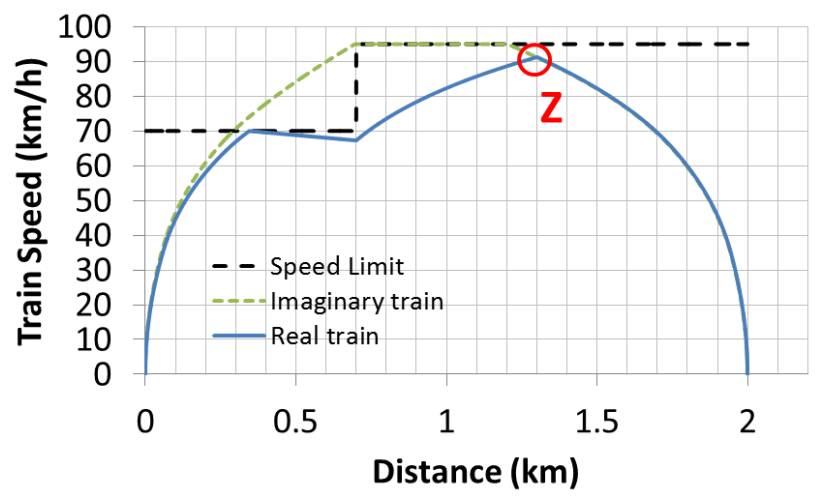

(b) Different track speed limits

Fig. 7. The proposed method to find the correct starting braking point under different track gradients and speed limits using the imaginary train.

\section{Study of energy saving effects by using numerical simulation}

In this chapter, the energy saving effects of the proposed regenerative brake notch is studied by using numerical simulation. To compare properly with the conventional constant deceleration brake, the maximum speed for both running profiles is assumed same as $95 \mathrm{~km} / \mathrm{h}$. In addition, the running time of constant deceleration brake is adjusted by changing its deceleration so that coincide with the running time of the regenerative brake notch. This is because the maximum speed and the running time are important factors of the energy consumption (Miyatake, 2011).

\subsection{Energy saving effects against running distance}

In this section, the energy saving effects against running distance is studied to reveal that the regenerative brake notch is effective in any range of running distance. Energy consumption between regenerative brake notch and constant deceleration brake is compared. The assumed running distances of each running pattern are $2 \mathrm{~km}, 3 \mathrm{~km}$ and $4 \mathrm{~km}$. The corresponding running time are set as $116 \mathrm{~s}, 157 \mathrm{~s}$ and $202 \mathrm{~s}$. To model the loss, constant efficiency of 0.85 is used as the total efficiency from the wheels to the pantograph. The results of these comparisons are shown in Fig. 8.

Figure 8 shows that the energy saving by the proposed regenerative brake notch are expected at each running distance, where the shorter running distance results in more energy saving. At shorter running distance, the vehicle speed at the starting braking point is higher than the case of longer running distance, because their maximum speeds are identical. Therefore, more energy is consumed by the mechanical brake in case of shorter distance. In addition, in the longer running distance, the coasting time is also longer. Thus, the energy consumed by the auxiliary loads increases the energy consumption. Figure 8 shows that $21 \%$ of energy saving is achieved at maximum under the given conditions in this study.

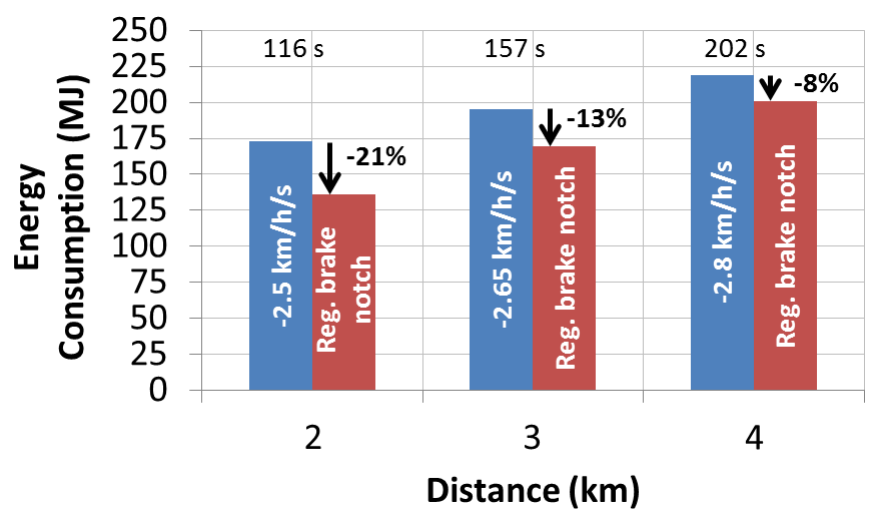

Fig. 8. Comparison of the energy consumption between the regenerative brake notch and the constant deceleration brake under the conditions of different running distance.

\subsection{Energy saving effects against braking point}

In the ATO system, brake is applied automatically at the correct position. However, in this regenerative brake notch system, the train driver has to apply the brake notch manually. The TICS can only inform the driver where the correct 
starting braking point is. Therefore, there could be variation of starting braking point. In this section, the energy saving effects against variation of braking point is studied to reveal that the proposed TICS-based information system is sufficiently accurate to save the energy. If the train speed is assumed as $90 \mathrm{~km} / \mathrm{h}$ when the brake is applied, one second delay of the driver's manipulation causes $25 \mathrm{~m}$ braking point variation from its correct position. If the brake is applied $25 \mathrm{~m}$ in advance to the correct position, it is able to avoid using the mechanical brake because the driver reduces the brake force to stop the train at the station. Therefore, only the case of $25 \mathrm{~m}$ closer to the station or the brake is delayed for 1 second should be discussed.

Figure 9(a) shows the running profile when the starting braking point is delayed $25 \mathrm{~m}$ or the brake is applied 1 second later. In Fig. 9(b), it is shown that the position of the real train is $25 \mathrm{~m}$ after the imaginary train. If the train runs under this profile, then the train will stop several meters after the station. Thus, the driver's manipulation by applying constant deceleration rate is required, for example at $140 \mathrm{~m}$ before the station as in Fig. 9(b). It is assumed that the driver applies $-3.0 \mathrm{~km} / \mathrm{h} / \mathrm{s}$ deceleration rate in low speed region to stop the train at correct position.

Figure 10 shows the energy consumption in the cases of Figs. 2 and 8. In Fig. 10, the regenerative brake notch with 1 second delay (number 3 from left) has 3\% less energy saving effect that the regenerative brake notch with proper braking timing (number 2 from left). Even so, $18 \%$ of energy saving can be expected compared to the constant deceleration brake (number 1 from left), which is ineligible energy saving effect.

The running time with 1 second delayed braking case $(115 \mathrm{~s})$ is reduced 1 second compared to the correct one (116 s) because stronger brake force is applied in low speed region to stop the train. In the right side of Fig. 10, the constant deceleration brake of $-2.7 \mathrm{~km} / \mathrm{h}$ and the 1 second delayed brake are compared to eliminate the effect of running time. Regenerative brake notch achieves $20 \%$ reduction of the consumption energy even the brake application is delayed for 1 second. Therefore, the proposed TICS-based information system is sufficiently accurate to save energy using the regenerative brake notch method.

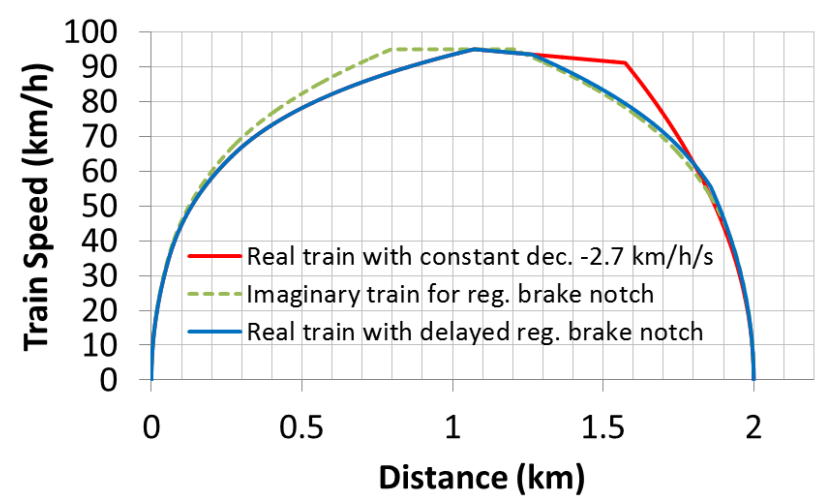

(a) Speed vs. distance

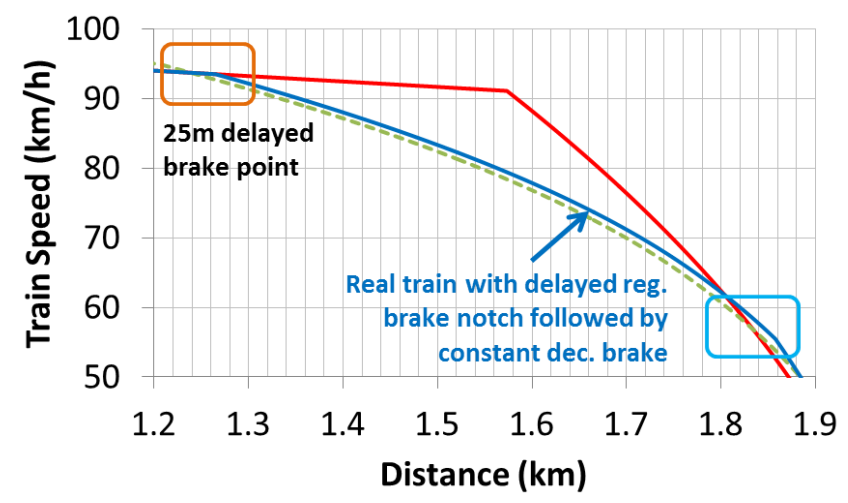

(b) The real train with delayed regenerative brake notch

Fig. 9. Comparison of the vehicle speed profiles between regenerative brake notch and constant deceleration brake in the case of $25 \mathrm{~m}$ delay of the braking point under the same running time.

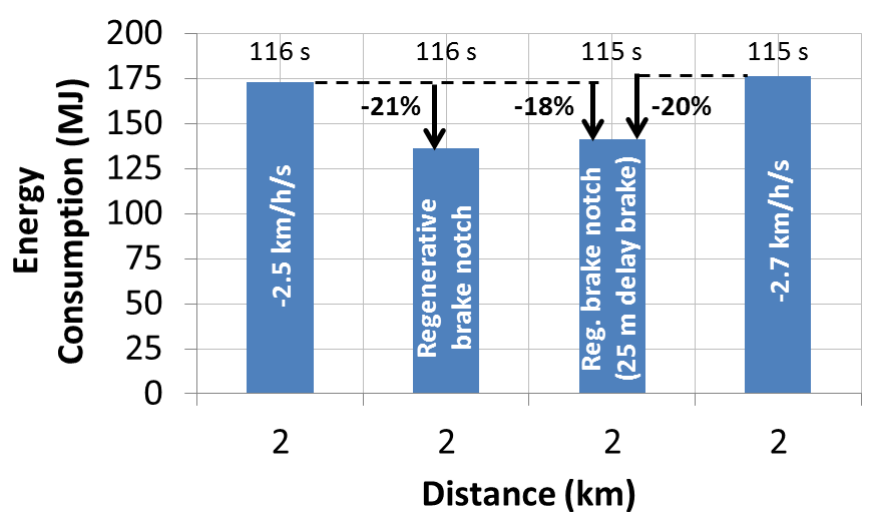

Fig. 10. Comparison of energy saving effects between the case of correct starting braking point and the case of $25 \mathrm{~m}$ delayed from starting braking point.

\subsection{Energy saving effects against slippery condition}


In the slippery condition, stopping the train at the station has more priority than saving the energy. The driver may apply the constant deceleration brake notch instead of the regenerative brake notch to secure the train stopping position. However, to gain more energy saving even at slippery rail condition, the use of regenerative brake notch should be considered. In this section, the effect of applying the regenerative brake notch for several slippery rail conditions is discussed.

The information that the train is running on slippery rail can be obtained from the traction motor controller in the inverter, for example through slip flag signal obtained by checking the acceleration rate of the wheel or the average motor current, which can be obtained within few seconds once the train starts running. However, it is difficult to obtain the information of how much the adhesion ratio in real time is. Therefore, this study assumes to use 5\% of adhesion coefficient when the slip flag is received by TICS from the traction motor controller regardless the actual adhesion coefficient, which is sufficiently low compared to $8.5 \%$ of adhesion coefficient as the design value of the maximum brake force in this study. In addition, this study assumes uniform adhesion coefficient along the rail between two stations. The energy saving effect of these assumptions to different actual adhesion coefficient will be discussed.

The adhesion coefficient $(\mu)$ is defined as the ratio between traction force per powered wheel to the axle load, which is given as follows:

$$
\mu=\frac{m \cdot a}{m \cdot g}=\frac{a}{g}
$$

At slippery rail conditions, the braking force must be limited according to the adhesion coefficient, which is shown in Fig. 11. For simplicity, the powering profile is not adjusted by assuming that re-adhesion control of the train works properly, and we pay more attention on the braking force reduction at slippery condition. In addition, the maximum running time of the train at the same running distance is decided by the adhesion coefficient. Thus, we cannot expect the same running time at different adhesion coefficient. In this case, the effect of regenerative brake notch is compared to the constant deceleration brake at the same running time. The results for constant deceleration brake to show the maximum running time at each adhesion coefficient are shown in Figs. 12(a) and 12(b). From these results, longer running time is required for lower adhesion coefficient.

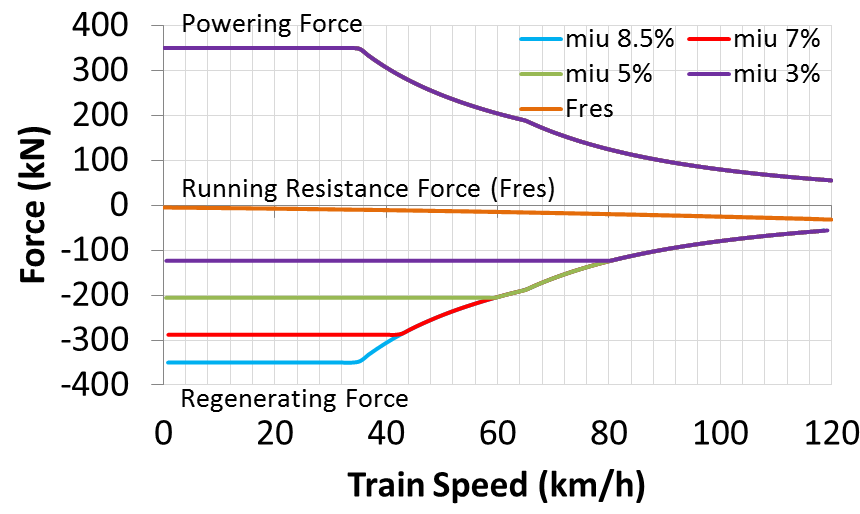

Fig. 11. Powering force and regenerating force characteristics at different adhesion coefficient.

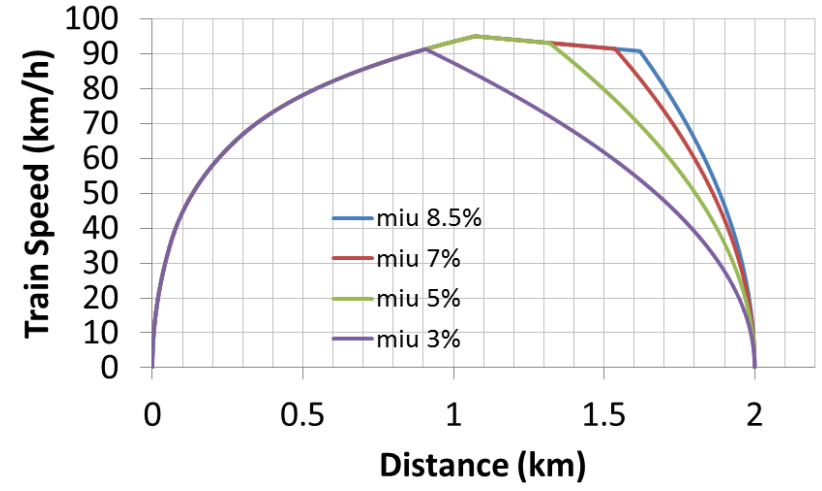

(a) Speed vs. distance

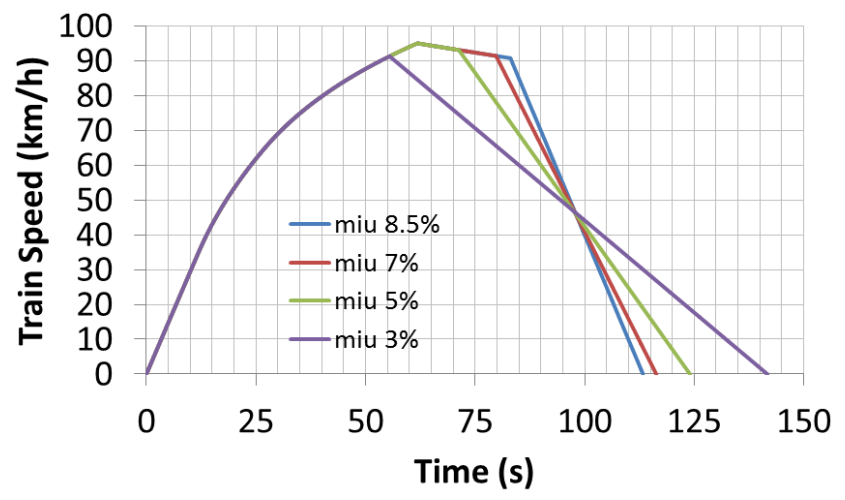

(b) Speed vs. time

Fig. 12. Comparison of the vehicle speed profile for constant deceleration brake at different adhesion coefficient. 


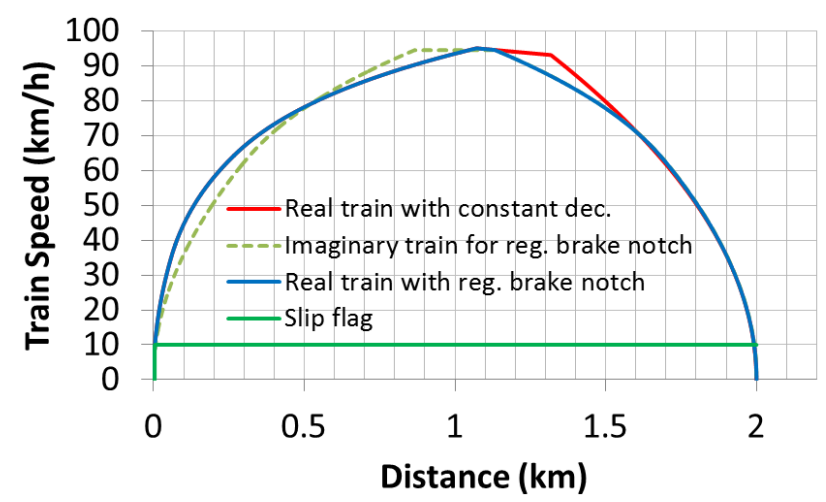

(a) Speed vs. distance

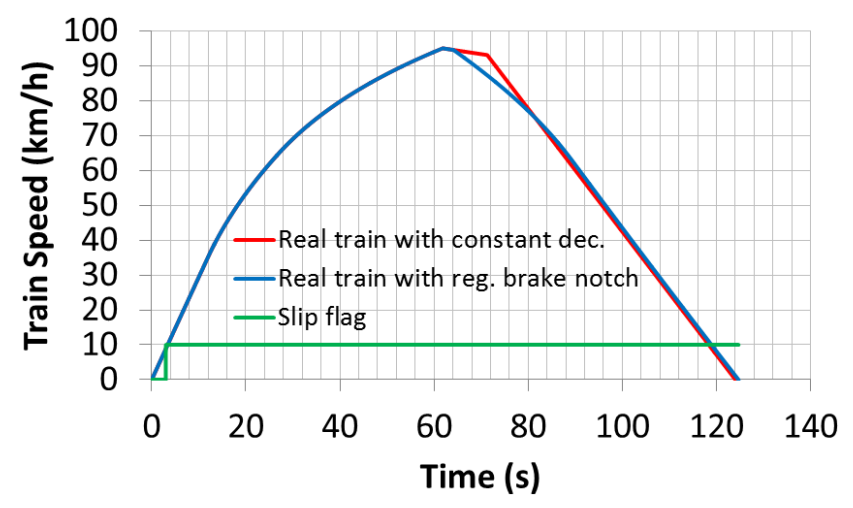

(b) Speed vs. time

Fig. 13. Comparison of the vehicle speed profiles between the regenerative brake notch and constant deceleration brake under $5 \%$ of adhesion coefficient

The comparison results between the regenerative brake notch and constant deceleration brake for the assumed 5\% of adhesion coefficient is shown in Fig. 13. The imaginary train is calculated according to the assumed adhesion coefficient once the slip flag information is received by the TICS, which is assumed around 3 seconds from the starting running time. Figure 13 shows that in the braking part, since the correct starting braking point is found successfully by comparing the running profile of imaginary train, the regenerative brake notch can be applied solely to stop the train at $5 \%$ of adhesion coefficient.

Since the actual adhesion coefficient varies depend on the rail condition, the assumed 5\% of adhesion coefficient may have error in estimating the correct starting braking point and the energy saving effect may deteriorate. The comparison of energy saving effect between the assumed adhesion coefficient and the actual one, i.e. $7 \%$ and $3 \%$, are given in Fig. 14. In this case, the slip flag is assumed known when the train starts running for fair comparison. As seen from Fig. 14, by applying weaker brake force in the low adhesion coefficient, the energy consumption becomes lower, but the running time is longer.

In case if the actual adhesion coefficient is higher than the assumed one, the starting braking point is given earlier than expected. In this case, the train can still run and stop at station using regenerative brake notch only, but the running time is slightly longer, e.g. $8 \mathrm{~s}$ in the case between 7\% and 5\% in Fig. 14. For lower actual adhesion coefficient than the assumed one, the regenerating force becomes higher than allowed. The re-adhesion control of the train may adjust the regenerating force automatically. In this case, it is difficult to apply the regenerative brake notch method, where the mechanical brake is required to stop the train. In addition, the regenerative brake notch method requires higher vehicle speed to start the braking, because the deceleration rate is lower than the case of constant deceleration brake. Thus, for lower adhesion coefficient, i.e. lower than 5\% in Fig. 11, it is difficult to apply the regenerative brake notch only to stop the train with the same running time at constant deceleration brake. Under this condition, the driver may judge which brake method is better to stop the train properly, either using the regenerative brake notch or the constant deceleration brake. The energy saving effect in the case of 3\% of adhesion coefficient in Fig. 14 means that the regenerative brake notch cannot be applied under this condition.

The energy saving effect for both cases has significant difference with the case of the assumed adhesion coefficient. Even so, the energy consumption for all cases is almost equal. For example, higher starting braking speed of 5\% adhesion case results in longer braking time compared to $7 \%$ adhesion case, which generally results in lower energy consumption. However, because the regenerating force of 5\% adhesion case is lower than that of $7 \%$ adhesion case, the energy consumption becomes almost equal. The same principle can be applied for $3 \%$ of adhesion coefficient. Thus, since the energy consumption for all adhesion coefficient cases is almost equal, the assumed 5\% of adhesion coefficient is sufficient to be used at any slippery condition. Therefore, the proposed regenerative brake notch is also effective to reduce the energy consumption at the slippery rail condition. 


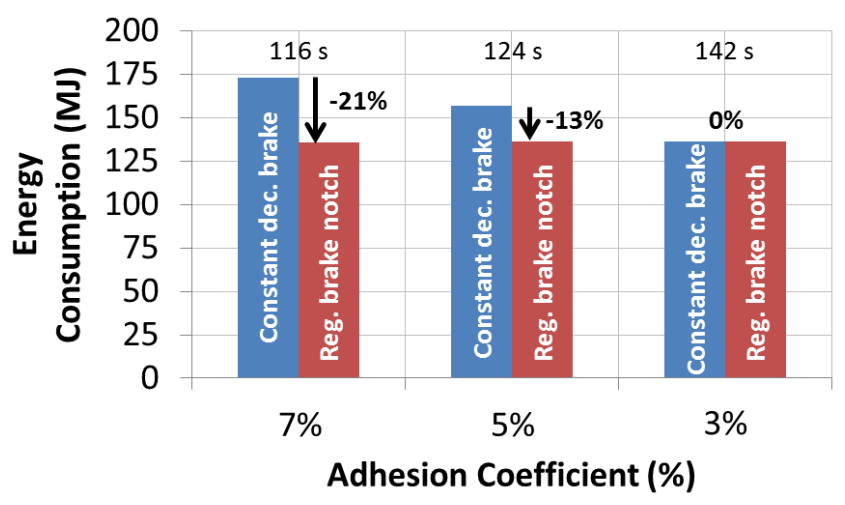

Fig. 14. The comparison of energy saving effect among different adhesion coefficients.

\section{Conclusions}

To reduce the energy losses by mechanical brake in the high speed region, the regenerative brake notch system that generates brake torque command equal to the torque-speed characteristics of the traction motors is proposed in this paper. The energy saving effect is studied by using the numerical simulation. This system achieves $21 \%$ reduction of the energy consumption at maximum under the given conditions.

The authors also propose a train information control system (TICS) based information system to notify the driver where the correct starting braking point is, because it is difficult to find the starting braking point manually by the driver. The imaginary train is used to find the starting braking point by estimating the braking pattern of real train during the powering phase. The merit of the proposed system is easy to get the value of speed and position of the train without the need to store a lot of data in terms of the initial braking speed and distance beforehand.

The effect of braking point variation is studied because this brake is applied not automatically but manually by the train driver. Even in the late braking case, $18 \%$ reduction of energy consumption is achieved by this system. In addition, the energy saving effect is also studied for different slippery rail condition. Even though the energy saving effect is different, but the energy consumption among different adhesion coefficient is almost equal. At the assumed adhesion coefficient, $13 \%$ reduction of energy consumption is obtained.

Therefore, these results show that the regenerative brake notch method and TICS-based information system is effective to save the energy of the train operation.

\section{References}

Koseki, T. and Noda, T., Run-Curve Design for Energy Saving Operation in a Modern DC-Electrification: Efficiency Calculation Assuming Perfect Regenerative Braking, Journal of Mechanical Systems for Transportation and Logistics, Vol. 3, No. 1 (2010), pp. 349-357.

Miyatake M., A Simple Mathematical Model for Energy-saving Train Scheduling, IEEJ Transactions on Industry Applications, Vol. 131, No. 6 (2011), pp. 860-861 (in Japanese).

Ogihara, T., Kodera, T. and Matsuo, H., Development of an Energy-Saving Light Rail Vehicle Powered by Large Lithium Ion Battery, Journal of Power and Energy Systems, Vol. 5, No. 3 (2011), pp. 295-301.

Suzuki, T., Hayashiya, H., Yamanoi, T. and Kawahara, K., Introduction and Practical Use of Energy Storage System with Lithium-ion Battery for DC Traction Power Supply System, IEEJ Journal of Industry Applications, Vol. 5, No. 1 (2016), pp. 20-25.

Watanabe, S., Yang, Z., Koseki, T., Kondo, K., Mizuma, T. and Hamazaki, Y., Experimental Verification of Power-Limiting Brake: Energy-Saving Train Operation Assistance, IEEJ Transactions on Industry Applications, Vol. 134, No. 8 (2014), pp. 767-775 (in Japanese). 